\title{
Studies on Key Technologies for Purchasing Management System Based on Application Service Provider
}

\author{
Zhou Guixian 1, a * \\ ${ }^{1}$ Information Institute GuiZhou College of Financial and Economic, Guiyang 550004, China \\ a120362538@qq.com
}

Keywords: application service provider (ASP); purchasing management; system structure; supply chain management

Abstract. This paper studied the base framework of purchasing management system based on application service provider (ASP), presented the system structure for purchasing management system based on application service provider (ASP), built the operation model of purchasing management system based on ASP, based on this research and study, built the main model of workflow. Finally, this paper projected the prototype of purchasing management system based on ASP, and realized some application of it.

\section{Introduction}

Networked manufacturing ASP platform system research, development and demonstration of the application of our country is a very important problem of small and medium enterprises. At home and abroad to business operation of the ASP model and procurement network resource sharing in the research to a certain extent, has obtained certain achievements [1].

But at present domestic most of the procurement system in essence is aimed at a single enterprise to provide services through the network, not on a rental basis based on ASP model system for multiple enterprises. And the cost of enterprise set up information platform is very high.

Draw lessons from the thought of the ASP model, this paper puts forward a network of procurement resources sharing mode based on ASP model, and based on this model designed to provide small and medium-sized enterprises including software outsourcing, technical support, training and a series of ASP service online. The platform includes procurement resources sharing information system, resources information system from supplier, contract and task management system, mail system, CA authentication system.

\section{The procurement network system based on ASP model}

In the current domestic more than 250 manufacturing enterprises, small and medium-sized enterprises accounted for more than $90 \%$, These small and medium-sized enterprises, while operating in good condition, but its information technology ability is weak, the low level of informationization. In the procurement, production and processing, management and operation mode, the small and medium-sized enterprises have great similar, but independent again, ones. So there is a mutual cooperation, mutual benefit cooperation space, also exist for these small and medium-sized enterprises provide the possibility of ASP service and ASP value-added services.

The discretion of the purchasing price and the purchasing cost directly affects the enterprise the cost of the product, and to leverage affects the overall efficiency and competitiveness of the enterprise. ASP procurement system is for the provision of small and medium-sized manufacturing enterprises procurement business cooperation to support a business model [2].

A hierarchical structure should be established in network manufacturing systems, which comprise basic layers, platform core layers, application framework layers, layers for professional applications, interface layers, and application system layers, as illustrated in Figure 1 


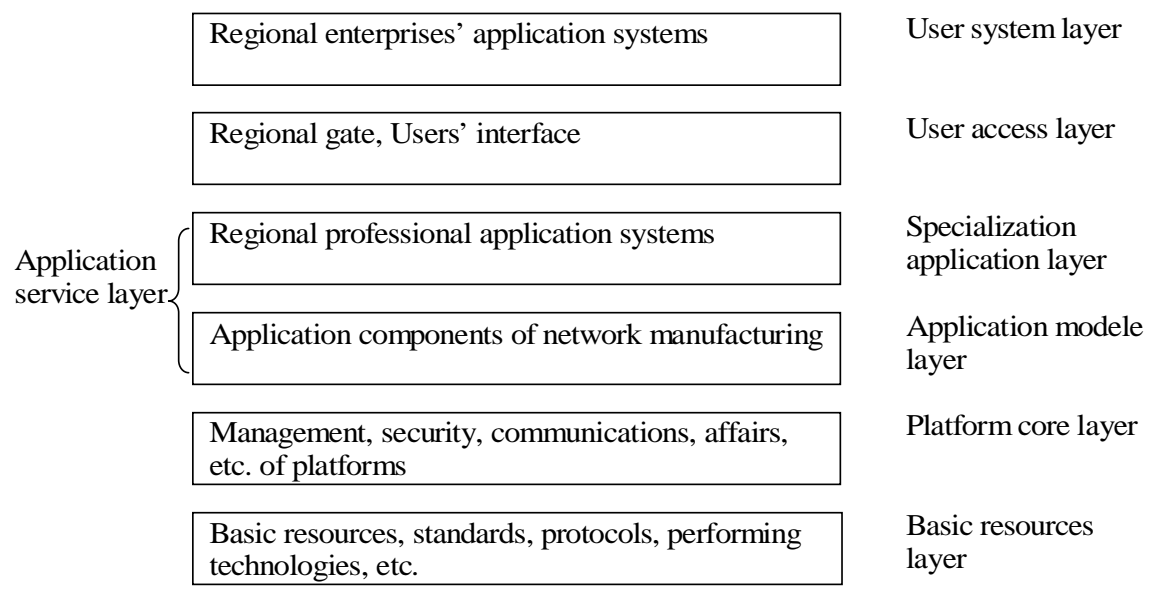

Figure 1 Regional network manufacturing system structure

(1) Basic layer: This layer acts as the base at the lowest level necessary to network manufacturing systems, consisting of storage of resources, databases, knowledge database, relevant standards and protocols, and relative enabling technologies, etc.

(2) Platform core layer: Most of services are provided for the users through the platform core layer. Needed in any network manufacturing systems, this layer supplies an assurance to stable performance of the systems, comprised of tools used for management, support, development, communication, and database services, and so on.

(3) Application component layer: This is a key layer through which network manufacturing services are provided to manufacturing enterprises and it includes the application components by which the network manufacturing ASP services are available to manufacturers at different locations. These application components can be further used for all areas or industries or for specific ones.

(4) Professional application layer: This layer, which is created by professional application systems to meet needs of different areas or industries, provides regional manufacturing enterprises with network manufacturing services, such as manufacturing resources management systems for auto parts manufacturers. The major difference between this layer and application component layer is that the former creates full solutions of the application system for areas or industries, while the latter the solutions of the components or the function class.

\section{The key technology of platform construction}

ASP procurement service can be divided into two types:

(1) the ASP procurement management platform, including software and hardware platform, companies hire purchase management platform to establish its own procurement management system, responsibility by the enterprise purchasing management. This type of service provides only a application system outsourcing service functions, ASP is responsible for the custom for the enterprise purchasing management platform, and is responsible for the procurement management platform of investment, construction, daily maintenance and management system platform, ASP and at the same time to provide professional business consulting, technical training and other services; Clients hire ASP platform of procurement management, pay the rent, is responsible for all the procurement business [3].

(2) the ASP provides the whole procurement services: enterprise only need to tell the ASP need what, how, when, the highest price, quality, etc., by the ASP combination similar procurement requirements of each enterprise collective procurement, for the cooperation of enterprise to obtain lower procurement costs and material purchasing price. Two types of ASP service of the business model is different. This kind of service type provides value-added services, provides outsourcing services. ASP is responsible for the procurement of value-added service platform of investment, construction, daily maintenance and management system platform, value-added business and is responsible for the procurement, ASP and at the same time to provide professional business consulting, technical training and other services; Customer to issue purchase requisitions and purchase to the ASP 
application to ASP to consolidate the purchasing requisition, centralized organization procurement tender, distribution of procurement, procurement task, the final pay the ASP business services.

This paper mainly discusses the second ASP service type of procurement system function framework and key technologies.

Platform construction is based on the author's research unit design architecture of ASP platform, therefore the platform design of data and communication protocol to follow various protocols of this platform, this article mainly from the procurement platform system function framework and key technologies are discussed.

The system itself is composed of sourcing and procurement services platform:

I the procurement business services include: trust relationship management, procurement requirements management, purchasing goods and materials distribution and other business services.

I Procurement service platform include: system management, membership management, supplier management, product information management, procurement directory management, purchasing plan management, pricing strategy management, other platform management. The software of the system based on J2EE architecture and the embedded Linux system.

Based on CORBA, WEB, software Agent encapsulation technology

Agent provides a new calculation and problem solving. CORBA specification provides a development of object-oriented distributed application framework. All according with the application of the interface, is easily integrated into CORBA and WEB system.

Using CORBA, WEB, Agent for the application software package has the following features:

I WEB technology to provide users with a unified user interface

I WEB Agent can blur the user task request is decomposed into some definite CORBA service invocation, further blocked application service implementation details.

I Between WEB server and the CORBA client adds a layer of the communication between the Agent and is conducive to the expansion of the system.

I CORBA Agent can provide information about the application server, such as traffic billing, conflict resolution, etc.

\section{Certificate authority and digital certificate}

In the network manufacturing model, the manufacturing business is not an isolated entity but a kind of usable manufacturing resource in a dynamic manufacturing environment, involved in the dynamic alliance construction. The allied businesses are distributed and dynamic, which leads to the following requirements for the ASP system in the security inspection:

(1) The allied businesses are distributed. The ASP platform should support remote access and can carry out user ID certification.

(2) The allied businesses are dynamic, among which some are relatively static. The ASP platform should provide flexible user identification mechanism in order to support the dynamic changes of the users.

(3) The identities of the allied members in different synergistic stage may have some change, so the ASP platform should support dynamic change of the user authority.

(4) The ASP-based network manufacturing processing is multi-user interactive and synergistic, so the ASP platform should provide an appropriate mechanism to support the inter-user communication.

(5) In the synergistic operation, for the purpose of obligation acknowledgement, it is necessary to have user's digital signature.

(6) The system also requires that the certification information transmission is encrypted and the information integrity during transmission is ensured.

By the construction of the ASP-based areas CA certification system, the ASP-based network manufacturing dynamic alliance can be formed and the synergic certification platform be built up, as is illustrated in Figure 2. Meanwhile, the ASP system service provider can also give fundamental security 
services for the businesses in other applications, such as emails, websites, office automation, and etc., the additional services the service provider can supply. The areas CA certification system provides the following digital certification services: personal ID certificate, server ID certificate, business certificate, secure Website certificate, personal email certificate, and encoded signature.

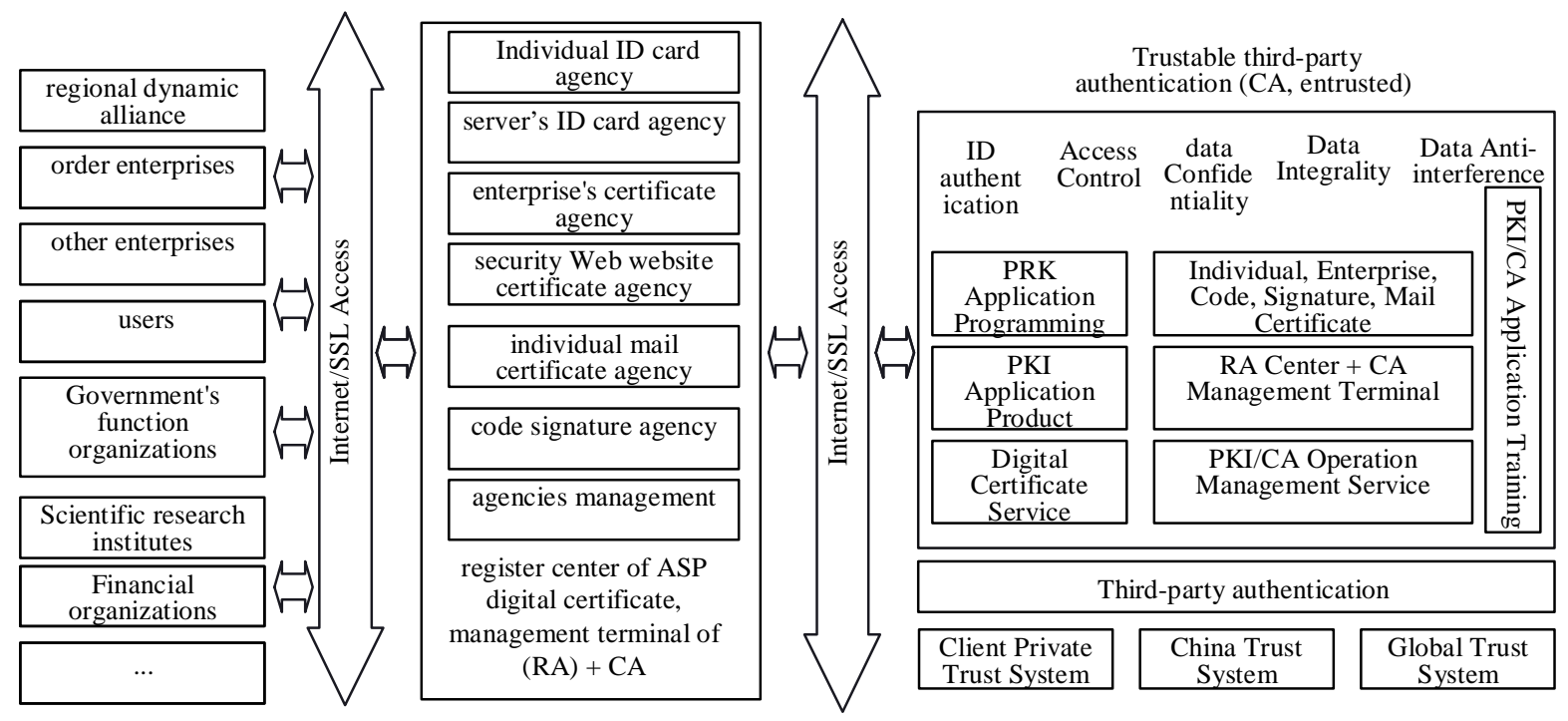

Figure 2 ASP-based CA platform application model

\section{Summary}

With the rapid development of global manufacturing industry, network manufacturing system will find a wider application. Procurement system based on the ASP model design, the author has carried out a lot of information collection, as well as the development of prototype system of research. Analysis and design of the procurement system based on ASP platform, is a kind of new purchasing for the current small and medium enterprises to explore, to carry out demonstration application of oriented to networked manufacturing ASP platform. ASP through establishing effective purchasing management, supply chain planning through effective stimulating plan implement demand chain procurement process, thus speeding up the turnover of enterprise procurement, realize the JIT (just-in-time) procurement strategy [4]. Reducing supply chain inventory, reduce the backlog of funds, increase the agility of supply chain, so as to improve the competitiveness of the supply chain.

\section{References}

[1] Qingsheng Xie, 2003, Study of The Network Manufacturing Model and Development Strategy in Chinese Enterprises, Digital Manufacture Science, Vol.1, No. 1, pp. 142 152

[2] Qingsheng Xie, 2003, The Model and Development Strategy of ASP. Manufacturing Information Engineering of China, Vol.32, No.1, pp. 66 70

[3] Qingsheng Xie, Yin Jian, November 2005, Study On Manufacture Resource Evaluating System Of the ASP-based Platform , IPROMS 2005 Proceedings

[4] Li Shaobo, Xie Qingsheng. The Framework Studies of Dynamic Alliance Manufacturing Resource Management System Based on Application Service Provider [J]. China Mechanical Engineering, 2005, 16(6):502-507 